\title{
Gobernabilidad en tiempos de crisis: la relación entre el Ejecutivo y el Legislativo en la gestión del presidente Nayib Bukele en el Salvador
}

\section{Governance in times of crisis: Executive and legislative relationship in the management of President Nayib Bukele in El Salvador}

\section{Mónica Tobar}

Fundación Salvadoreña para el Desarrollo Económico y Social

mtobar@fusades.org

\section{Resumen}

La segunda transición de poder político en el Ejecutivo representa una oportunidad y un reto para el desarrollo institucional en El Salvador. El periodo de construcción democrática, iniciado en 1992, ha permitido el desarrollo paulatino de la institucionalidad salvadoreña. En los últimos 30 años se han realizado esfuerzos importantes para institucionalizar el uso de canales democráticos para la toma de decisiones. El análisis retoma la interacción entre el Gobierno del presidente Nayib Bukele y el Órgano Legislativo durante su primer año de Gobierno, con el objetivo de ofrecer una valoración sobre las condiciones de gobernabilidad y la capacidad del sistema político salvadoreño para solventar las diferencias entre actores politicos mediante canales democráticos.

Palabras clave: Gobernabilidad, democracia, diálogo, El Salvador, Nayib Bukele.

\begin{abstract}
The second political transition of power in El Salvador poses a challenge for the democratic institutions. The aftermath of the Peace Accord, signed in 1992, has allowed the gradual development of the Salvadoran democratic institutions. In the past 30 years, significant efforts have been made to institutionalize the use of democratic channels for decision-making. This article tackles the interaction between the government of President Nayib Bukele and the Legislative Assembly, in order to explore the strength of democratic governance in the Salvadoran political system.
\end{abstract}

Keywords: Democratic, governance, dialogue, El Salvador, Nayib Bukele.

Articulo: Recibido el 31 de mayo de 2020 y aprobado el 26 de junio de 2020

\section{Cómo citar este artículo:}

Tobar, M. (2020). Gobernabilidad en tiempos de crisis: la relación entre el Ejecutivo y el Legislativo en la gestión del presidente Nayib Bukele. Reflexión política 22(45), pp. 70-79. doi: https://doi.org/10.29375/01240781.3919

\section{Introducción}

El desarrollo democrático en El Salvador es un proceso vivo y complejo. La historia política del país está marcada por el conflicto armado que inició en 1980 y culminó con la firma del Acuerdo de Paz el 16 de enero de 1992. Uno de los principales legados de este acuerdo es la transformación de las instituciones políticas y electorales como cimientos del proceso de transición hacia la democracia. 
El Acuerdo sentó las bases para la democratización (Holiday y Stanley, 1993) y contempló transformaciones en el sistema electoral, así como la reestructuración de las fuerzas de seguridad pública, lo que devolvió la subordinación de estas al poder civil. Por otra parte, la capacidad de consenso demostrada por los actores políticos involucrados, lo posicionan como el ejercicio más relevante en materia de diálogo democrático hasta la fecha.

Luego de más de 30 años de la suscripción de este Acuerdo, el desarrollo democrático ha permitido dos momentos de alternancia en el Ejecutivo, así como la institucionalización de canales democráticos para la toma de decisiones y su posterior implementación. La llegada al poder del presidente Nayib Bukele representa un nuevo capítulo en la historia política del país. Con su triunfo, finalizó con tres décadas de Gobierno de dos fuerzas políticas que ocuparon la presidencia de la República desde la firma del pacto de Chapultepec. De acuerdo con lo anterior, resulta relevante analizar la respuesta institucional ante las decisiones del mandatario y sus repercusiones sobre las condiciones de gobernabilidad en El Salvador.

Este artículo se centra en la interacción entre el Gobierno del presidente Nayib Bukele y el Órgano Legislativo durante su primer año de Gobierno. El documento no pretende hacer una revisión exhaustiva de los indicadores de gobernabilidad sino, más bien, ofrece un aporte sobre las reacciones institucionales frente a las decisiones del nuevo Ejecutivo, con el objetivo de analizar la capacidad del sistema político salvadoreño para solventar las diferencias a través de los canales institucionales.

En la primera y segunda sección se presentan, respectivamente, una breve reseña del contexto político salvadoreño y de los elementos que facilitaron la llegada al poder del presidente Bukele, y el marco analítico para el abordaje de la gobernabilidad. El tercer apartado examina la relación que han mantenido el Ejecutivo y el Legislativo durante el primer año de Gobierno. Finalmente, se ofrecen algunas reflexiones preliminares sobre las respuestas institucionales a los desencuentros entre ambos, y de qué manera estas diferencias afectan la gobernabilidad.

\section{Nayib Bukele y la segunda alternancia}

El 3 de febrero de 2019, Nayib Bukele, candidato de la fórmula presidencial del partido Gran Alianza por la Unidad Nacional (GANA), fue electo como presidente de la República de El Salvador para el periodo 2019-2024. El triunfo electoral terminó con 30 años de alternancia en el Ejecutivo entre los partidos Alianza Republicana Nacionalista (ARENA) y el Frente Farabundo Martí para la Liberación Nacional $(\text { FMLN })^{1}$, dos de los principales actores del proceso de transición democrática en El Salvador, que desde 1994 concentraron más del 85\% de los votos válidos en los comicios presidenciales (FUSADES, 2019a).

El presidente Bukele obtuvo el 50.3\% de los votos válidos con una participación electoral del 51.88\% (Tribunal Supremo Electoral, 2019). De acuerdo con Rodríguez y Solano (2020), estos resultados muestran una disminución de la participación equivalente a nueve puntos porcentuales, en relación con la segunda vuelta de la elección presidencial de 2014. A pesar de esta reducción, es notable la victoria electoral del presidente Bukele en todos los departamentos del país.

El porcentaje de participación sugiere que la introducción de un nuevo participante en la contienda no fue suficiente para ampliar la base de votantes, más bien, el candidato capturó la preferencia de ciudadanos que respaldaban otros institutos políticos. En esta línea, los resultados de una encuesta de panel sobre la intención de voto en las elecciones presidenciales de 2019, Córdova, Argueta y Erazo (2020) identifican como uno de los elementos asociados a la preferencia por el partido GANA, el rechazo de los ciudadanos hacia los partidos mayoritarios (ARENA y FMLN). Asimismo, el estudio permitió identificar cambios en la intención de voto entre dos comicios presidenciales (2014 y 2019), lo que respalda la hipótesis de la migración de votantes desde los partidos tradicionales hacia el nuevo participante en contienda.

${ }^{1}$ En 2009 el FMLN protagonizó la primera alternancia en el Ejecutivo con la elección del expresidente Mauricio Funes Cartagena. 
La llegada de Nayib Bukele a la presidencia se caracterizó por una campaña intensiva en el uso de medios digitales, la construcción de una narrativa de antipolítica que lo diferenciaba de los partidos tradicionales y el uso de estrategias polémicas para la inscripción de su candidatura. Con respecto a este último punto, cabe mencionar que el actual presidente ha militado en tres institutos politicos: el FMLN, partido del cual fue expulsado; Cambio Democrático (CD); y GANA, este último partido le permitió optar por la jefatura de Gobierno.

El proceso de inscripción de la candidatura de Nayib Bukele como aspirante a la presidencia estuvo marcado por su expulsión del FMLN, partido por el cual fue electo como alcalde en dos ocasiones; la imposibilidad de oficializar su candidatura con el partido Nuevas Ideas, que no logró cumplir los plazos de inscripción para participar en los comicios de 2019 y la cancelación de Cambio Democrático por no alcanzar el umbral de votos requeridos por Ley en la elección legislativa de 2015 (Tribunal Supremo Electoral, 2018).

El desgaste progresivo de los partidos políticos que gobernaron el país en la etapa de construcción democrática, así como la prevalencia de brechas en la satisfacción de las demandas básicas de la ciudadanía ${ }^{2}$, perfilaron un escenario favorable para la concreción de las aspiraciones políticas del actual presidente. Los datos del Barómetro de las Américas (Análisis preliminar del Barómetro de las Américas de LAPOP, 2018) muestran que el 62\% de las personas salvadoreñas manifestaron apoyo a la democracia como forma de Gobierno, el 28.8\% confiaba en los partidos políticos, mientras que el $40.8 \%$ consideró que El Salvador no es una democracia. Este último dato refleja una brecha importante en cuanto al reconocimiento de este sistema político y la apropiación de los valores democráticos como parte de la identidad nacional.

La insatisfacción de los ciudadanos con los partidos políticos tradicionales le abrió un nicho al actual mandatario para posicionarse como opción presidencial. Durante los meses de campaña electoral, el entonces candidato construyó un discurso en el que se distanciaba de la política tradicional y se configuraba como la única alternativa viable (Sáenz, 2020; FUSADES, 2019a). Un elemento importante durante la contienda fue la exposición de casos de corrupción, que involucraban a líderes políticos de los partidos mayoritarios, situación que reforzó la narrativa mesiánica del aspirante a la presidencia.

El uso intensivo de las redes sociales y la agresiva estrategia para capturar el voto joven fue otro distintivo de la campaña de Nayib Bukele (FUSADES, 2019b). Sin embargo, investigaciones posteriores demostraron que el candidato invirtió más recursos en posicionarse a través de medios tradicionales como la televisión con el fin de ampliar su base de simpatizantes (Acción Ciudadana, 2019). Un aspecto para destacar es que, a pesar de ser un candidato joven, y de no haber realizado campañas a nivel territorial, obtuvo el respaldo de todos los grupos etáreos.

El 1 de junio de 2019, Nayib Bukele fue juramentado como presidente de la República de El Salvador. La incursión de GANA como partido de Gobierno, conlleva desafíos para la gobernabilidad debido a la modesta participación de este instituto político en la Asamblea Legislativa. En este sentido, Artiga (2019) advirtió que la falta de respaldo legislativo del presidente Bukele podría incrementar la conflictividad entre los partidos políticos, lo que dificulta la construcción de consensos para la toma de decisiones.

\section{Gobernabilidad en tiempos de transición}

El periodo de construcción democrática, iniciado en 1992, ha permitido el desarrollo paulatino de la institucionalidad salvadoreña. Si bien existen déficits importantes en la capacidad del sistema político para resolver las tensiones que puedan ocasionarse entre los actores, en los últimos 30 años se han realizado esfuerzos importantes para institucionalizar el uso de canales democráticos para la toma de decisiones y su posterior implementación.

La segunda transición de poder político en el Ejecutivo ${ }^{3}$ representa una oportunidad y un reto para el desarrollo democrático en El Salvador. La incorporación de nuevos actores al sistema político puede oxigenar el funcionamiento de la contraloría horizontal y fortalecer la capacidad institucional para solventar las diferencias, pero, de forma simultánea, este proceso depende de la solidez del entramado institucional para la gestión del poder político.

${ }^{2}$ De acuerdo a la Dirección General de Estadísticas y Censos (Encuesta de Hogares de Propósitos Múltiples 2019, 2020), en 2019 el $23.48 \%$ de los hogares salvadoreños se encontraban en situación de pobreza.

${ }^{3}$ La primera transición ocurrió en 2009 con la victoria del FMLN, después de 20 años de Gobierno del partido ARENA. 
La gobernabilidad democrática implica el respeto y el funcionamiento de dos principios básicos: la existencia de procesos establecidos para la toma de decisiones y el desconocimiento inicial de los actores sobre los resultados de las deliberaciones. Asimismo, la gobernabilidad está sujeta a la existencia de límites, económicos y normativos, para la atención de las demandas ciudadanas, los cuales determinan la solidez de las instituciones y la eficacia de implementación de las políticas (Sojo, 2003, p. 154).

La gobernabilidad también puede ser entendida como el equilibrio entre las demandas de los actores estratégicos y la capacidad del Gobierno para responderlas de manera eficiente, como resultado de procedimientos democráticos, institucionalizados $\mathrm{y}$ en un proceso donde los puntos de vista de los actores son considerados. La gobernabilidad supone estabilidad política, pero también apela a la existencia de instituciones con capacidades necesarias para abordar colectivamente los retos y oportunidades (Moreno, 2004).

Las altas expectativas de la ciudadanía respecto al nuevo Gobierno, sumadas a los elevados niveles de aprobación del presidente (IUDOP, 2020), incrementan la presión para dar respuesta a las necesidades de la población, situación que se agudizó con el impacto de la crisis sanitaria y económica derivada de la pandemia por la COVID-19. Los desencuentros entre Órganos de Estado complican o impiden la ejecución de las decisiones, tensionando el sistema político.

Artiga (2007) define la gobernabilidad como la "capacidad del sistema político para adoptar decisiones vinculantes y ponerlas en práctica" (p. 236), a través de las instituciones encargadas de la toma de esas decisiones y su posterior implementación. En este sentido, este artículo retoma la conceptualización de este autor para examinar las reacciones institucionales del Legislativo y el Ejecutivo durante los primeros meses de gestión del presidente Bukele.

Las reacciones, sociales o institucionales, influyen en la toma de decisiones políticas y son una parte fundamental de la gobernabilidad, ya que afectan directamente la capacidad para implementar estas decisiones. No obstante, los desacuerdos interorgánicos son parte del ejercicio cotidiano de los frenos y contrapesos de cualquier sistema democrático, en caso de que no sean canalizados por la vía institucional; es decir, haciendo uso de los mecanismos constitucionales para solventar las discrepancias, y no sean reconocidos como legitimos por los actores involucrados, erosionan la gobernabilidad, en la medida en que obstaculizan la capacidad para ejecutar las decisiones.

\section{Confrontación interorgánica: la relación entre el Ejecutivo y el Legislativo (2019- 2020)}

El trabajo coordinado entre los Órganos del Estado da forma al proceso de toma de decisiones políticas. El Ejecutivo requiere del respaldo de los legisladores para la aprobación del marco legal necesario para implementar y financiar las políticas públicas; mientras que el Órgano Legislativo, materializa sus decisiones mediante las diferentes carteras del Gobierno ${ }^{4}$. En este sentido, la dinámica entre estos actores debe tener como base la búsqueda de consensos que permitan responder efectivamente a las demandas de la ciudadanía.

La dinámica entre el Gobierno y los legisladores se enmarca en el respeto al Estado de derecho y el cumplimiento de sus respectivas obligaciones constitucionales. En un sistema democrático la existencia de desacuerdos no se traduce en la ruptura de los canales de diálogo para la construcción de acuerdos, estas diferencias deben ser gestionadas a través de los mecanismos institucionales diseñados para este propósito. No obstante, el deficiente desarrollo de la institucionalidad democrática amplifica el espacio para la imposición de procesos unilaterales para la toma de decisiones que afectan a la sociedad, los cuales se acentúan en períodos de crisis.

La toma de posesión del nuevo presidente transformó la dinámica legislativa y reconfiguró la correlación política para la aprobación de las iniciativas gubernamentales. Las dificultades para establecer acuerdos multipartidarios, apoyados por el Ejecutivo, están vinculadas, principalmente, con cuatro factores: a) la débil participación del partido oficial en la Asamblea Legislativa; b) la toma de decisiones arbitrarias y unilaterales por parte del presidente; c) el discurso de deslegitimación del Ejecutivo hacia los partidos tradicionales; y d) la poca capacidad de diálogo y consenso entre los actores involucrados en la toma de decisiones.

${ }^{4}$ Si bien se reconoce la importancia del control institucional, ejercido por el Órgano Judicial, para efectos del presente análisis, la revisión se acota a la relación entre el Ejecutivo y el Legislativo. 
Cuadro 1. El Salvador: representación y alianzas legislativas del partido GANA (2012-2020)

\begin{tabular}{ccccc}
\hline \multirow{2}{*}{ Período } & $\begin{array}{c}\text { Representación } \\
\text { legislativa }\end{array}$ & \% del total $^{\mathrm{a}}$ & Peronismo Kirchnerista & Alianzas legislativas \\
\cline { 3 - 4 } & & GANA & ARENA+FMLN & \\
\hline $2012-2015$ & 11 escaños & $13 \%$ & $76.1 \%$ & FMLN/PCN (58.3\%) \\
\hline $2015-2018$ & 11 escaños & $13 \%$ & $70.2 \%$ & FMLN/PCN (57.1\%) \\
\hline $2018-2021$ & 10 escaños & $11.9 \%$ & $71.4 \%$ & ARENA/PCN/PDC (67\%)
\end{tabular}

aLa Asamblea Legislativa está conformada por 84 diputados.

Fuente: Artiga (2019) y FUSADES (2017;2018 y 2019).

El partido oficial (GANA), que nació de una fisura en el partido ARENA, ha mantenido magros resultados en los comicios legislativos desde su incursión en 2012, y cuenta con una modesta representación de legisladores, por lo que requiere del acompañamiento de al menos uno de los partidos mayoritarios para impulsar la agenda gubernamental (Cuadro 1).

Pese a las diferencias ideológicas, los legisladores de GANA acompañaron las propuestas del FMLN durante sus dos períodos al frente del Ejecutivo (2009-2014 y 2014-2019), junto con el Partido de Concertación Nacional (PCN). Esta alianza facilitó la aprobación de iniciativas polémicas, algunas declaradas inconstitucionales por la Sala de lo Constitucional (SCN) de la Corte Suprema de Justicia. El patrón de votación conjunta se diluyó con la llegada de Nayib Bukele a la jefatura del Ejecutivo. En el primer año de administración del presidente se observa una tendencia hacia la conformación de un nuevo arreglo legislativo, integrado por ARENA, GANA, PCN y el Partido Demócrata Cristiano (PDC). Este giro ha facilitado el acompañamiento legislativo de los requerimientos gubernamentales.

La llegada al poder de un actor externo a los partidos que gobernaron El Salvador desde la firma de la Paz, aunado al impacto de la crisis económica y sanitaria, consecuencia de la pandemia por de la COVID-19, suponen un shock simultáneo para el sistema político salvadoreño que introduce presión sobre las condiciones de gobernabilidad y pone a prueba el uso de los canales democráticos para la toma de decisiones.

En el caso salvadoreño, el diseño institucional le otorga al presidente poderes legislativos limitados. Si bien el mandatario participa activamente en el proceso de formación de Ley, se requiere de la coordinación con otros Órganos de Estado para la toma de decisiones vinculantes. Las facultades constitucionales del mandatario son: a) la iniciativa de ley, a través de sus ministros; b) sancionar, promulgar y publicar las leyes y ejecutarlas; c) vetar proyectos de ley por razones de inconstitucionalidad o inconveniencia u observarlos para corregir ciertos aspectos de forma; d) influir en la designación de funcionarios que le compete a la Asamblea Legislativa; y e) proponer, a través del Consejo de Ministros, la suspensión de garantías constitucionales y convocar extraordinariamente a la Asamblea Legislativa cuando los intereses de la República lo demanden y siempre que se cumplen determinados presupuestos establecidos en la Ley Fundamental y en sentencias de la Sala de lo Constitucional (Constitución de la República de El Salvador, 1983; Artiga, 2007).

En este sentido, la emisión de vetos es un indicador de gobernabilidad, en la medida que expresa el rechazo del presidente a las decisiones de los legisladores ${ }^{5}$. El ritmo de aplicación de estos mecanismos ofrece una lectura sobre la imposibilidad de construir acuerdos sólidos para responder a las demandas de la población. En su primer año de Gobierno, Bukele vetó 23 iniciativas

${ }^{5}$ En El Salvador el veto presidencial es absoluto, es decir, no se contempla el veto parcial de las iniciativas. El mecanismo puede sustentarse en la inconveniencia o inconstitucionalidad del decreto aprobado. En este último caso, la superación del veto se realiza por mayoría calificada, o sea, con dos tercios de los votos legislativos. 
-10 de estas fueron ratificadas posteriormente por los legisladores-. El ritmo de aplicación de este mecanismo es considerablemente mayor a lo documentado en los últimos 30 años, lo cual refleja la conflictividad entre ambos Órganos de Estado (Cuadro 2).

Cuadro 2. El Salvador: vetos emitidos por quinquenio (1989-2020)

\begin{tabular}{ccc}
\hline Presidente & $\begin{array}{c}\text { Periodo de } \\
\text { Gobierno }\end{array}$ & Número de vetos \\
\hline $\begin{array}{c}\text { Alfredo } \\
\text { Cristiani }\end{array}$ & $1989-1994$ & 1 \\
\hline $\begin{array}{c}\text { Armando } \\
\text { Calderón Sol }\end{array}$ & $1994-1999$ & 4 \\
\hline $\begin{array}{c}\text { Francisco } \\
\text { Flores }\end{array}$ & $1999-2004$ & 59 \\
\hline $\begin{array}{c}\text { Antonio Saca } \\
\text { Mauricio } \\
\text { Funes }\end{array}$ & $2004-2009$ & 0 \\
\hline $\begin{array}{c}\text { Salvador } \\
\text { Sánchez Cerén }\end{array}$ & $2014-2019$ & 10 \\
\hline $\begin{array}{c}\text { Nayib Bukele } \\
\text { a }\end{array}$ & $2019-2024$ & 23
\end{tabular}

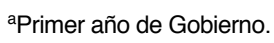

Fuente: FUSADES (2020b) con información de la Asamblea Legislativa y la Presidencia de la República.

Las principales áreas de desencuentro son las reformas de las disposiciones aplicables a los comicios legislativos y municipales de 2021 y la gestión de la emergencia por de la COVID-19. La primera dimensión, contempló 5 vetos presidenciales a las siguientes iniciativas: el voto por rostro para los candidatos a alcaldes, la aplicación del voto desde el exterior -vetado en dos ocasiones-, el incremento a las sanciones a funcionarios por la falta de actualización del registro electoral y la prohibición de propaganda en el transporte colectivo (FUSADES, 2020b). En cuanto al manejo de la crisis sanitaria, el presidente emitió 8 vetos a decretos que regulaban la atención de la emergencia, 5 de los cuales fueron superados por los legisladores. Este último punto será retomado en secciones posteriores.

El uso de la antipolítica, presente durante la campaña presidencial, se consolidó como narrativa oficial y se acompañó de excesos en el ejercicio del poder y de un continuo desconocimiento de las decisiones de las instituciones. Desde el inicio del período de gestión del presidente Bukele, la relación entre el Ejecutivo y el Legislativo se ha caracterizado por la recurrencia de episodios de confrontación y la adopción de un discurso de descalificación hacia el Órgano Legislativo y los partidos políticos tradicionales.

A pesar de las diferencias, el presidente contó con el respaldo de los diputados para la aprobación del presupuesto nacional, la deuda pública y las principales iniciativas, sometidas a consideración del pleno (FUSADES, 2020b). Sin embargo, el discurso confrontativo del gobernante sugiere que estos resultados no responden a una estrategia de diálogo y consenso político, sino, a la creación constante de escenarios de conflicto como medida de presión para acelerar la aprobación de iniciativas. Esta práctica se caracteriza por el establecimiento de una narrativa binaria sobre el ejercicio del poder, en la cual las diferencias políticas se reducen a una dualidad entre los adeptos del Gobierno y las personas que se oponen al bienestar de los ciudadanos.

La escalada de tensión entre el presidente y los legisladores se acentuó con dos hechos en particular: la militarización de la Asamblea Legislativa el 9 de febrero de 2020 y el abordaje de la emergencia nacional ocasionada por la pandemia de la COVID-19. Ambos escenarios han desencadenado crisis de gobernabilidad, en tanto que se registra una ruptura de los canales democráticos para solventar las diferencias interorgánicas.

El 9 de febrero de 2020, el presidente Bukele irrumpió en la Asamblea Legislativa, acompañado de las fuerzas de seguridad - Fuerza Armada de El Salvador (FAES) y la Policía Nacional Civil-, como medida de presión para acelerar la aprobación de un préstamo destinado a financiar el Plan Control Territorial, su apuesta más importante en materia de seguridad. En los días previos a la militarización del recinto legislativo, el mandatario convocó, a través de su Consejo de Ministros, a una sesión legislativa extraordinaria, dedicada a la aprobación del empréstito, pese a que el pleno se encontraba sesionando de manera normal (Sáenz, 2020).

El mantenimiento de la subordinación de la FAES al poder civil es uno de los principales logros del Acuerdo de Paz, por lo que el apoyo militar para la usurpación legislativa sienta un precedente negativo en cuanto al abuso de la fuerza como estrategia de presión política. Este acontecimiento 
modificó la relación interorgánica y derivó en la activación de mecanismos de control legislativo, como la creación de una comisión especial para la investigación de los hechos y la deducción de responsabilidades, así como la interpelación del Ministro de la Defensa Nacional, pero la sesión fue pospuesta indefinidamente debido a la emergencia por la pandemia de la COVID-19 (López, 2020).

\subsection{Reacciones institucionales en el marco de la emergencia por la COVID-19}

A partir de marzo de 2020, los esfuerzos institucionales en El Salvador se volcaron a atender la crisis sanitaria por la pandemia de la COVID-19. La actitud autoritaria del presidente Bukele, materializada en el irrespeto a la separación de poderes y al Estado de derecho en meses previos, generó un ambiente de inestabilidad política que permeó en el manejo de la crisis y afectó la dinámica entre el Ejecutivo y el Legislativo.

Durante las primeras semanas de la emergencia, los legisladores construyeron acuerdos multipartidarios para regular las medidas de confinamiento y para decretar el estado de emergencia nacional, así como la supresión de garantías constitucionales concretas, en aras de disminuir la circulación de personas y la posibilidad de contagios. Desde el inicio de la emergencia, el Ejecutivo optó por la solicitud de autorización a los legisladores para ampliar sus atribuciones constitucionales para la gestión de la pandemia.

La adopción de medidas excepcionales es "la respuesta más común en las democracias constitucionales para legitimar las decisiones tomadas para hacer frente a la crisis sanitaria" (Freidenberg, 2020, p. 19-20). Sin embargo, las ventajas obtenidas por el Ejecutivo, están supeditadas a la deliberación $\mathrm{y}$ al respaldo legislativo, como único mecanismo para otorgar y revertir facultades extraordinarias a los gobernantes.

En El Salvador, la adopción temprana de medidas de confinamiento y cierre de fronteras contó con el respaldo inicial del Órgano Legislativo. No obstante, se han registrado constantes desencuentros en torno a la necesidad de prolongar las facultades extraordinarias otorgadas al Ejecutivo para gestionar la emergencia ante las reiteradas denuncias de irregularidades y excesos en el uso de la fuerza pública para la aplicación de las medidas de confinamiento.

El Ejecutivo ha adoptado la toma de decisiones unilaterales como principal estrategia para el manejo de la crisis. En el marco de la pandemia, se emitieron 9 de los 21 vetos presidenciales registrados en el primer año de

Cuadro 3. El Salvador: principales puntos de disputa entre el Ejecutivo y el Legislativo en el marco de la emergencia por de la COVID-19

\begin{tabular}{cccc}
\hline $\begin{array}{c}\text { Mecanismo } \\
\text { de control }\end{array}$ & Área & Cantidad & $\begin{array}{c}\text { Respuesta } \\
\text { legislativa }\end{array}$ \\
\hline & $\begin{array}{c}\text { Estado de } \\
\text { emergencia }\end{array}$ & 1 & 1 veto superado \\
\cline { 2 - 4 } & $\begin{array}{c}\text { Derechos } \\
\text { Humanos }\end{array}$ & 5 & $\begin{array}{c}4 \text { vetos } \\
\text { superados } \\
\text { Veto pendiente } \\
\text { de discusión }\end{array}$ \\
\cline { 2 - 4 } & $\begin{array}{c}\text { Aspectos } \\
\text { económicos } \\
\text { y fiscales }\end{array}$ & 2 & $\begin{array}{c}1 \text { veto } \\
\text { superado }\end{array}$ \\
\cline { 2 - 4 } & $\begin{array}{c}\text { Medidas de } \\
\text { reactivación } \\
\text { económica }\end{array}$ & 1 & $\begin{array}{c}1 \text { veto } \\
\text { superado }\end{array}$
\end{tabular}

Fuente: Elaboración propia con base en información oficial de la Presidencia de la República de El Salvador.

Gobierno y 5 de estos fueron superados (Cuadro 3).

Los principales puntos de desencuentro se relacionan con la aprobación de decretos que procuraban la defensa de derechos fundamentales, como la seguridad social y laboral, la protección del personal de salud y el establecimiento de protocolos para el retorno de salvadoreños atrapados en el exterior (Asamblea Legislativa, 2020). Los vetos del presidente a iniciativas que procuraban la protección de la ciudadanía es consistente con el patrón de denuncias de violaciones a los derechos humanos ante diferentes instituciones de control ${ }^{6}$.

Las dificultades para la aprobación de iniciativas ley en un contexto de emergencia nacional denotan una limitada capacidad para el diálogo y consenso, tanto en la etapa de toma de decisiones, como en su ejecución. En el caso de los vetos que han sido superados por los legisladores, el marco legal salvadoreño contempla que la SCN dirima la validez de las medidas; sin embargo, la

${ }^{6}$ Durante el período de emergencia la SCN recibió 142 procesos de hábeas corpus. 32 amparos y 15 demandas de inconstitucionalidad (CSJ, 2020), en su mayoría relacionados con la vulneración del derecho a la salud e información en los centros de contención gubernamental, diseñados para albergar a las personas provenientes del extranjero- sujetas a un periodo de cuarentena obligatoria de 30 días contados desde su ingreso a El Salvador. Estos recintos también fueron utilizados por el Gobierno para el confinamiento de ciudadanos que incumplieron la cuarentena domiciliar durante el periodo de recintos también fuero
emergencia nacional. 
necesidad de solventar el conflicto mediante la justicia constitucional sugiere la incapacidad de las instituciones encargadas para el cumplimiento de sus funciones. Asimismo, la poca claridad sobre las normas vigentes para regular la cuarentena domiciliar y la reapertura de la actividad productiva refleja el impacto que tiene la distorsión en el proceso de toma de decisiones sobre la capacidad, tanto de instituciones como de ciudadanos para asumirlas y ejecutarlas.

\section{Reflexiones finales}

El sistema político salvadoreño enfrenta el reto de gestionar, a través de canales institucionales, los conflictos entre los Órganos de Estado. Si bien el ejercicio de la contraloría horizontal ha resultado positivo, la confrontación prolongada entre el Ejecutivo y el Legislativo dificulta la construcción de acuerdos para la atención de la emergencia y plantea interrogantes sobre el nivel de desarrollo del sistema democrático.

La evolución de la tensa relación entre el Ejecutivo y Legislativo indica la ruptura de los canales institucionales para la gestión de las diferencias políticas. El presidente Bukele ha privilegiado la toma de decisiones unilaterales para evadir el control político, el diálogo y la negociación. En este sentido, la distorsión del proceso de toma de decisiones, como consecuencia del rechazo a los canales institucionales, y sus interlocutores, para la gestión de las diferencias, es un espacio fértil para la escalada de actitudes de corte autoritario que, de no ser limitadas efectivamente por el sistema de control institucional, pondrían en riesgo los avances realizados en materia democrática.

La interacción entre la Asamblea y el Gobierno durante el primer año de gestión del presidente Bukele, revela la prevalencia de brechas en la capacidad institucional para facilitar la construcción de consensos. La falta de acuerdos entre estos actores genera incertidumbre sobre el carácter vinculante de las decisiones y erosiona su legitimidad de cara a la ciudadanía.

Finalmente, la falta de capacidad de diálogo socava la institucionalidad y dilata la toma de decisiones críticas para responder a las demandas de la población, particularmente en contextos de crisis. La persistencia de relaciones de confrontación entre Órganos de Estado y la normalización de la toma de decisiones, fuera del proceso establecido en el marco constitucional, genera un nicho peligroso para el ascenso de actitudes autoritarias en el ejercicio del poder, cuya institucionalización supondría un grave retroceso para el desarrollo democrático del país.

\section{Referencias}

Acción Ciudadana. (2019). Elección presidencial 2019. Monitoreo de la Propaganda Electoral. San Salvador: Centro de Monitoreo de Transparencia y Democracia.

Artiga, Á. (2007). Gobernabilidad y democracia en El Salvador. San Salvador: UCA Editores.

Artiga, Á. (2019). Análisis político de las elecciones presidenciales: El Salvador 2019. San Salvador: Heinrich Böll Stiftung.

Asamblea Legislativa. (15-27 de Mayo de 2020). Asamblea Legislativa República de El Salvador. Obtenido de Archivo Histórico: https://www.asamblea.gob.sv/ index.php/

Córdova, R., Argueta, C. y Erazo, M. (2020). La población salvadoreña en las elecciones presidenciales 2019: Análisis de la intención de voto mediante un Panel Electoral. San Salvador: Fundación Dr. Guillermo Manuel Ungo.

CSJ. (2020). Boletín Informativo 21 de abril de 2020. San Salvador: Corte Suprema de Justicia.

DIGESTYC. (2020). Encuesta de Hogares de Propósitos Múltiples 2019. Ciudad Delgado: Dirección General de Estadísticas y Censos.

Freidenberg, F. (2020). La gestión política de la pandemia COVID-19: tensiones y oportunidades. En N. González, M. Marván y G. Salmorán, Emergencia sanitaria por COVID-19 (págs. 17-28). Ciudad de México: Instituto de Investigaciones Jurídicas, Universidad Autónoma de México.

FUSADES. (2005). Las Instituciones Democráticas en El Salvador: Valoración de Rendimientos y Plan de Fortalecimiento. San Salvador: Fundación Salvadoreña para el Desarrollo Económico y Social.

FUSADES. (2018). Diálogo democrático y políticas públicas en El Salvador. Antiguo Cuscatlán: Fundación Salvadoreña para el Desarrollo Económico y Social.

FUSADES. (2019a). El Salvador. Año político. Junio de 2018-mayo de 2019. San Salvador: Fundación Salvadoreña para el Desarrollo Económico y Social.

FUSADES. (2019b). Desagregando la participación electoral. Elecciones presidenciales. Antiguo Cuscatlán: Fundación Salvadoreña para el Desarrollo Económico y Social.

FUSADES. (2020a). El Salvador. Año Político junio 2019- 
mayo 2020. Antiguo Cuscatlán: Fundación Salvadoreña para el Desarrollo Económico y Social.

FUSADES. (2020b). Algunas reflexiones sobre el COVID-19, el desempeño de los sistemas políticos y la democracia. Antiguo Cuscatlán: Fundación Salvadoreña para el Desarrollo Económico y Social.

Holiday, D. y Stanley, W. (1993). Building the Peace: Preliminary Lessons from El Salvador. Journal of International Affairs, 415-438.

IUDOP. (2020). La población salvadoreña evalúa la situación general del país a finales del año 2019. Antiguo Cuscatlán: Instituto Universitario de Opinión Pública, Universidad Centroamericana José Simeón Cañas.

LAPOP. (2018). Análisis preliminar del Barómetro de las Américas de LAPOP. Vanderbilt University.

López, G. (27 de marzo de 2020). Escucharán declaraciones sobre los hechos ocurridos el 9 de febrero. Obtenido de Asamblea Legislativa de El Salvador: https://www.asamblea.gob.sv/node/10159

Moreno, M. (2004). Instituciones y gobernabilidad democrática: claves del círculo virtuoso del desarrollo. En M. (. Moreno, Gobernabilidad, instituciones y desarrollo. América Latina y Honduras (págs. 46-48). Tegucigalpa: Banco Interamericano de Desarrollo, Instituto Interamericano para el Desarrollo Social.

Rodríguez, L. M. y Solano, L. (2020). Las elecciones de 2018 y 2019 en El Salvador: ¿El ascenso de la tercera vía? En M. Alcántara, América Latina Vota 2017-2019. Madrid: Editorial Tecnos.

Sáenz, R. (25 de Febrero de 2020). Centroamérica, ¿adónde vas? Populismo y nuevos actores políticos. Obtenido de Política Exterior: https:// www.politicaexterior.com/centroamerica-adonde-vaspopulismo-nuevos-actores-politicos/

Sojo, C. (2003). In search of Democratic Governance in Central America. Journal of International Affairs, 57(1), 153-167.

Tribunal Supremo Electoral. (2018). Procedimiento de cancelación de inscripción del partido político Cambio Democrático (CD). Resolución definitiva. San Salvador: Tribunal Supremo Electoral.

Tribunal Supremo Electoral. (2019). Memoria de elecciones presidenciales 2019. San Salvador: Tribunal Supremo Electoral de El Salvador. 